Bull. Austral. Math. Soc.

VoL. 39 (1989) [397-404]

\title{
PROPERTY PRESERVING OPERATORS
}

Evelyn M. Silvia

Let $S$ denote the class of functions of the form $f(z)=z+\sum_{k=2}^{\infty} a_{k} z^{k}$ that are analytic and univalent in $|z|<1$. Given $f \in S$ and $a, b, c$, real numbers other than $0,-1,-2, \ldots$, let $\Omega(a, b, c ; f)=F(a, b, c ; z)^{\star} f(z)$ where $z^{-1} F(a, b, c ; z)=1+\sum_{k=1}^{\infty}\left((a)_{k}(b)_{k}\right) /\left((c)_{k}(1)_{k}\right) z^{k}$ is a hypergeometric Gauss function with $(a)_{0}=1$ and $(a)_{k}=a(a+1) \ldots(a+k-1)$ and * denotes the Hadamard product. For $q_{n}(z)=z+a_{2} z^{2}+\ldots+a_{n} z^{n}\left(a_{n} \neq 0, n=5,6\right)$ in $S$, it is shown that $\Omega\left(\gamma+1,1, \gamma+2 ; q_{n}\right)=\Phi_{\gamma}\left(q_{n}\right)=\left((\gamma+1) / z^{\gamma}\right) \int_{0}^{z} t^{\gamma-1} q_{n}(t) d t$, $\gamma>-1$, is univalent in $|z|<1$. This extends the result previously known for $n=3$ and $n=4$. Also, we obtain a necessary and sufficient condition involving $a, b$, and $c$ such that $\Omega(a, b, c ; \cdot)$ preserves the subclass of $S$ consisting of starlike functions of order $\alpha$, $0 \leqslant \alpha \leqslant 1$, with $a_{k} \leqslant 0$.

\section{INTRODUCTION}

Let $S$ denote the class of functions of the form

$$
f(z)=z+\sum_{n=2}^{\infty} a_{n} z^{n}
$$

that are analytic and univalent in $\Delta=\{z:|z|<1\}$, with $S^{\star}(\alpha), 0 \leqslant \alpha \leqslant 1$, designating the subclass of $S$ consisting of functions starlike of order $\alpha$. We shall denote by $T$ the subclass of $S$ consisting of functions that may be expressed in the form

$$
f(z)=z-\sum_{n=2}^{\infty} a_{n} z^{n}, \quad a_{n} \geqslant 0,
$$

and will set $T^{\star}(\alpha)=T \cap S^{\star}(\alpha)$. It is known [10] that $f \in T^{\star}(\alpha)$ if and only if its coefficients satisfy the inequality

$$
\sum_{n=2}^{\infty}(n-\alpha) a_{n} \leqslant(1-\alpha)
$$

Received $22 \mathrm{July}, 1988$

Copyright Clearance Centre, Inc. Serial-fee code: 0004-9729/89 \$A2.00+0.00. 
The class of polynominals of degree $n, q_{n}(z)=z+\sum_{k=2}^{n} a_{k} z^{k}, a_{n} \neq 0$, that are univalent in $\Delta$ will be designated by $P_{n}$. In the next section, we will consider the general integral operator

$$
\Phi_{\gamma}(f(z))=\frac{(\gamma+1)}{z^{\gamma}} \cdot \int_{0}^{z} t^{\gamma-1} f(t) d t \quad(\gamma>-1) .
$$

The Hadamard product or convolution of two power series

$$
f(z)=\sum_{n=0}^{\infty} a_{n} z^{n} \text { and } h(z)=\sum_{n=0}^{\infty} c_{n} z^{n}
$$

is defined as the power series

$$
(f \star h)(z)=\sum_{n=0}^{\infty} a_{n} c_{n} z^{n}
$$

For $G(z)=\sum_{n=1}^{\infty}((\gamma+1) /(\gamma+n)) z^{n}$ we note that $\Phi_{\gamma}(f(z))=(f \star G)(z)$. Since $G(z)$ is known [8] to be convex for $\gamma>0$, it follows from the work of Ruscheweyh and Sheil-Small [9] that $\Phi_{\gamma}(f), \gamma>0$, is close-to-convex or starlike of order $\alpha$ whenever $f(z)$ is such. It was shown in [12] that for $f \in T^{\star}(\alpha)$ we actually have $\Phi_{\gamma}(f(z)) \in T^{\star}((2+\alpha \gamma) /(3+\gamma-\alpha))$ which is a little better than we get from closure under convolution with a convex function.

The question of preservation of univalence under $\Phi_{\gamma}$ is still relatively open for discussion. In $[5]$ an example of an $f(z)$ univalent in $\Delta$ with $\Phi_{0}(f)$ not univalent is given. For $\gamma=1$, the radius of close-to-convexity for $S$ [4] assures the univalence of $\Phi_{\gamma}(f(z)), f \in S$, in $|z|<\rho$ where $0.80<\rho<0.81$. Whether $\rho$ can be replaced by 1 is still unknown. In [6], it is shown that if $f \in P_{n}$, then $\Phi_{0}(f)$ is univalent for $|z|<2 \sin (\pi / n)$ and $\Phi_{1}(f)$ is univalent for $|z|<2 \sin (\pi /(n+1))$. Hence, $\Phi_{0}$ preserves $P_{n}$ for $n \leqslant 6$ and $\Phi_{1}$ preserves $P_{n}$ for $n \leqslant 5$. Finally, from [11], we know that $\Phi_{\gamma}\left(P_{n}\right) \subset P_{n}$ for $n=3,4$ and for all $\gamma>-1$. In Section 2, we extend the latter result to $n=5$ and $n=6$. In Section 3 , we will consider a generalisation of the operator $\Phi_{\boldsymbol{\gamma}}$.

For $f \in S$, and $a, b, c$ real numbers other than $0,-1,-2, \ldots$, let

$$
\Omega(a, b, c ; f)=F(a, b, c ; z) \star f(z)
$$

where

$$
z^{-1} F(a, b, c ; z)=1+\sum_{k=1}^{\infty} \frac{(a)_{k}(b)_{k}}{(c)_{k}(1)_{k}} z^{k}
$$


is a hypergeometric Gauss function and $(a)_{0}=1,(a)_{k}=a(a+1) \cdots(a+k-1)$. Note that $\Omega(\gamma+1,1, \gamma+2 ; f)=\Phi_{\gamma}(f)$.

In [11], it was shown that for $q \in P_{3}$ and $c \geqslant|a|>0, \Omega(a, 1, c ; q) \in P_{3}$. Let $\Sigma_{k}(a, b, c ; z)$ denote the $k$ th partial sum of $F(a, b, c ; z)$. We know that $\Sigma_{2}(a, b, c ; z)$ is convex in $\Delta$ if and only if

$$
4|a||b| \leqslant|c|
$$

In [2], it is shown that for $f(z)=z+\beta z^{2}+\delta z^{3}, \beta, \delta$ real, and $0 \leqslant \delta \leqslant 1 / 15$, the condition $(1+9 \delta) / 4 \geqslant \beta \geqslant 8 \delta /(1+5 \delta)$ implies that $f$ is convex. Thus, $\Sigma_{3}(a, b, c ; z)$ is convex for $0 \leqslant(a)_{2}(b)_{2} /(c)_{2} \leqslant 2 / 15$ and

$$
\frac{2(c)_{2}+9(a)_{2}(b)_{2}}{8(c)_{2}} \geqslant \frac{a \cdot b}{c} \geqslant \frac{8(a)_{2}(b)_{2}}{2(c)_{2}+5(a)_{2}(b)_{2}} .
$$

It follows that $\Omega_{2}(a, b, c ; \cdot)$ and $\Omega_{3}(a, b, c ; \cdot)$ preserve the subsets of $P_{2}$ and $P_{3}$ consisting of functions that are convex, starlike of order $\alpha$ and close-to-convex as long as (2) and (3) are satisfied, respectively. In the last section, we obtain a necessary and sufficient condition involving $a, b$ and $c$ such that $\Omega(a, b, c ; \cdot)$ preserves the class $T^{\star}(\alpha)$.

\section{THE OPERATOR $\Phi_{\gamma}$}

In order to show that $P_{n}$ is preserved under $\Phi_{\gamma}$ for $n=5$ and $n=6$, we will use two lemmas.

LEMmA A. [11] For $q_{k}(z)=z+a_{2} z^{2}+\ldots+a_{k} z^{k} \in P_{k}$, a sufficient condition for $\Phi_{\gamma}\left(q_{k}\right)$ to be in $P_{k}$ is that the polynomial

$$
G_{k-1, \gamma}(z)=\sum_{j=0}^{k-1}\left(\begin{array}{c}
k-1 \\
j
\end{array}\right) \cdot\left[\frac{\gamma+1}{\gamma+k-j}\right] z^{j}
$$

have all of its zeros in $|z| \leqslant 1$.

LemmA B. [7] (Cohn's Rule) For $f(z)=a_{0}+a_{1} z+\ldots+a_{n} z^{n}$, let $f^{\star}(z)=$ $\bar{a}_{0} z^{n}+\bar{a}_{1} z^{n-1}+\ldots+\bar{a}_{n}$. Then, if $\left|a_{0}\right|<\left|a_{n}\right|$, the polynomial $f_{1}$ given by $z f_{1}(z)=$ $\bar{a}_{n} f(z)-a_{0} f^{\star}(z)$ has one zero less than $f$ has in $\Delta$.

Given a polynomial of degree $n$, as long as it is applicable, we can use Lemma $B$ successively $n-1$ times to obtain a first degree polynomial. It follows that if the zero of the first degree polynomial is in $\Delta$, then all $n$ zeros of the original polynomial lie in $\Delta$. Using this method we have: 
Theorem 1. For $2 \leqslant k \leqslant 6$, if $q_{k} \in P_{k}$, then $\Phi_{\gamma}\left(q_{k}\right) \in P_{k}$ for all $\gamma>-1$.

ProOF: For $k=2$, the result is trivial. The cases $k=3$ and $k=4$ were obtained earlier [11]. For $k=5$, from Lemma $\mathrm{A}$, it suffices to show that all the zeros of

$$
G(z)=\frac{\gamma+1}{\gamma+5}+4\left(\frac{\gamma+1}{\gamma+4}\right) z+6\left(\frac{\gamma+1}{\gamma+3}\right) z^{2}+4\left(\frac{\gamma+1}{\gamma+2}\right) z^{3}+z^{4}
$$

lie in $|z| \leqslant 1$. Since $(\gamma+1) /(\gamma+5)<1$, Lemma B applies and we can form

$$
z \bar{G}_{1}(z)=1 \cdot G(z)-\frac{\gamma+1}{\gamma+5} \cdot G^{\star}(z)
$$

from which we obtain

$$
\begin{aligned}
G_{1}(z) & =\frac{(\gamma+5)^{2}}{8(\gamma+3)} \cdot \tilde{G}_{1}(z) \\
& =\frac{(\gamma+1)(\gamma+5)}{(\gamma+2)(\gamma+4)}+3 \frac{(\gamma+1)(\gamma+5)}{(\gamma+3)^{2}} z+3 \frac{(\gamma+1)(\gamma+5)}{(\gamma+2)(\gamma+4)} z^{2}+z^{3} .
\end{aligned}
$$

For $\gamma>-1$, we have $(\gamma+1)(\gamma+5) /((\gamma+2)(\gamma+4))<1$. To apply Lemma B we form

$$
z \tilde{G}_{2}(z)=1 \cdot G_{1}(z)-\frac{(\gamma+1)(\gamma+5)}{(\gamma+2)(\gamma+4)} \cdot G_{1}^{\star}(z) .
$$

This leads to

$$
\begin{aligned}
G_{2}(z) & =\frac{(\gamma+2)^{2}(\gamma+4)^{2}}{3\left(2 \gamma^{2}+12 \gamma+13\right)} \cdot \tilde{G}_{2}(z) \\
& =\frac{(\gamma+1)(\gamma+5)\left(2 \gamma^{2}+12 \gamma+19\right)}{(\gamma+3)^{2}\left(2 \gamma^{2}+12 \gamma+13\right)}+4 \frac{(\gamma+1)(\gamma+2)(\gamma+4)(\gamma+5)}{(\gamma+3)^{2}\left(2 \gamma^{2}+12 \gamma+13\right)} z+z^{2} \\
& \equiv \mu+4 \lambda z+z^{2}
\end{aligned}
$$

Once again we have $\mu<1$ for $\gamma>-1$, so we let

$$
z \bar{G}_{3}(z)=1 \cdot G_{2}(z)-\mu \cdot G_{2}^{\star}(z)
$$

and obtain

$$
G_{3}(z)=\frac{1}{1-\mu^{2}} \cdot \tilde{G}_{3}(z)=\frac{4 \lambda}{1+\mu}+z .
$$

Now, since $0<\mu<1,4 \lambda /(1+\mu)<1$ if and only if

$$
\begin{aligned}
4(\gamma+1)(\gamma+2)(\gamma+4)(\gamma+5)< & (\gamma+3)^{2}\left(2 \gamma^{2}+12 \gamma+13\right) \\
& +(\gamma+1)(\gamma+5)\left(2 \gamma^{2}+12 \gamma+19\right)
\end{aligned}
$$


which is equivalent to $4\left(2 \gamma^{2}+12 \gamma+13\right)>0$. This last inequality is satisfied for $\gamma>-1$. Therefore, $G_{3}$ has one root in $\Delta$. Applying Lemma B sequentially, it follows that $G_{2}$ has 2 roots in $\Delta, G_{1}$ has 3 roots there and, finally, $G$ has all 4 roots in $\Delta$. Thus, by Lemma A, $\Phi_{\gamma}\left(P_{5}\right) \subset P_{5}$.

The process detailed for $k=5$ goes just as smoothly for $k=6$. To apply Lemma A, we consider

$$
H(z)=\sum_{j=0}^{5}\left(\begin{array}{l}
5 \\
j
\end{array}\right)\left[\frac{\gamma+1}{\gamma+6-j}\right] z^{j}
$$

We obtain the following finite sequence of auxiliary polynomials

$$
\begin{aligned}
H_{1}(z)= & \frac{(\gamma+1)(\gamma+6)}{(\gamma+2)(\gamma+5)}+4 \frac{(\gamma+1)(\gamma+6)}{(\gamma+3)(\gamma+4)} z+6 \frac{(\gamma+1)(\gamma+6)}{(\gamma+3)(\gamma+4)} z^{2} \\
& +4 \frac{(\gamma+1)(\gamma+6)}{(\gamma+2)(\gamma+5)} z^{3}+z^{4} \\
H_{2}(z)= & \frac{(\gamma+1)(\gamma+6)\left(\gamma^{2}+7 \gamma+14\right)}{(\gamma+3)(\gamma+4)\left(\gamma^{2}+7 \gamma+8\right)}+3 \frac{(\gamma+1)(\gamma+2)(\gamma+5)(\gamma+6)}{(\gamma+3)(\gamma+4)\left(\gamma^{2}+7 \gamma+8\right)} z \\
& +3 \frac{(\gamma+1)(\gamma+2)(\gamma+5)(\gamma+6)}{(\gamma+3)(\gamma+4)\left(\gamma^{2}+7 \gamma+8\right)} z^{2}+z^{3} \\
H_{3}(z)= & \xi+\xi z+z^{2} \quad \text { for } \xi=\frac{(\gamma+1)(\gamma+2)(\gamma+5)(\gamma+6)}{\gamma^{4}+14 \gamma^{3}+69 \gamma^{2}+140 \gamma+90}
\end{aligned}
$$

and

$$
H_{4}(z)=z+\frac{\xi}{1+\xi}
$$

Since $\gamma^{4}+14 \gamma^{3}+69 \gamma^{2}+140 \gamma+90=\frac{1}{2}(A+B)$ where

$$
A=(\gamma+3)(\gamma+4)\left(\gamma^{2}+7 \gamma+8\right)
$$

and

$$
B=(\gamma+1)(\gamma+6)\left(\gamma^{2}+7 \gamma+14\right),
$$

we know that $1 / 2(A+B)>0$ for $\gamma>-1$ and $\xi>0$. Also, $\xi<1$ if and only if

$$
(\gamma+1)(\gamma+2)(\gamma+5)(\gamma+6)<\left(\gamma^{4}+14 \gamma^{3}+69 \gamma^{2}+140 \gamma+90\right)
$$

which is equivalent to

$$
2\left(\gamma^{2}+14 \gamma+15\right)>0
$$

and is satisfied for $\gamma>-1$. Therefore, $\xi /(\xi+1)<1$. We conclude that $H_{4}$ has one root in $\Delta$ and $H$ has 5 roots there. 
Remarks 1. To see that the sufficient condition is not met for $k=7, \gamma>-1$, Lemma $B$ proves to be a bit unwieldy. Instead we can appeal to the Schur-Cohn Criteria [7]: If for the polynomial $f(z)=a_{0}+a_{1} z+\ldots+a_{n} z^{n}$, all the determinants

$$
\Delta_{k}=\left|\begin{array}{ccccccccc}
a_{0} & 0 & 0 & \ldots & 0 & a_{n} & a_{n-1} & \ldots & a_{n-k+1} \\
a_{1} & a_{0} & 0 & \ldots & 0 & 0 & a_{n} & \ldots & a_{n-k+2} \\
\vdots & \vdots & \vdots & \vdots & \vdots & \vdots & \vdots & \vdots & \vdots \\
a_{k-1} & a_{k-2} & a_{k-3} & \ldots & a_{0} & 0 & 0 & \ldots & a_{n} \\
\bar{a}_{n} & 0 & 0 & \ldots & 0 & \bar{a}_{0} & \bar{a}_{1} & \ldots & \bar{a}_{k-1} \\
\bar{a}_{n-1} & \bar{a}_{n} & 0 & \ldots & 0 & 0 & \bar{a}_{0} & \ldots & \bar{a}_{k-2} \\
\vdots & \vdots & \vdots & \vdots & \vdots & \vdots & \vdots & \vdots & \vdots \\
\bar{a}_{n-k+1} & \bar{a}_{n-k+2} & \bar{a}_{n-k+3} & \ldots & \bar{a}_{n} & 0 & 0 & \ldots & \bar{a}_{0}
\end{array}\right|
$$

for $k=1,2,3, \ldots, n$ are different from 0 , then $f$ has no zeros on the circle $|z|=1$ and $p$ zeros in this circle, where $p$ is the number of variations of sign in the sequence $1, \Delta_{1}, \Delta_{2}, \ldots, \Delta_{n}$. Thus in order for $f$ to have $n$ zeros in $\Delta$ the sequence must have alternating signs. For the case $k=7$ we consider

$$
F(z)=\sum_{j=0}^{6}\left(\begin{array}{l}
6 \\
j
\end{array}\right)\left(\frac{\gamma+1}{\gamma+7-j}\right) z^{j} .
$$

Then, for $\gamma>-1, \Delta_{0}=1, \Delta_{1}=(-12(\gamma+4)) /\left((\gamma+7)^{2}\right)<0$, and

$$
\Delta_{2}=\frac{720(\gamma+4)^{2}\left(2 \gamma^{2}+16 \gamma+19\right)}{(\gamma+2)^{2}(\gamma+6)^{2}(\gamma+7)^{4}}>0 .
$$

However,

$$
\Delta_{3}=\frac{345,600(\gamma+4)^{3}\left(\gamma^{2}+8 \gamma-3\right)\left(2 \gamma^{4}+32 \gamma^{3}+179 \gamma^{2}+408 \gamma+279\right)}{(\gamma+2)^{4}(\gamma+3)^{2}(\gamma+5)^{2}(\gamma+6)^{4}(\gamma+7)^{6}}
$$

is positive for $\gamma>-4+\sqrt{19}$. Thus, at least for $\gamma>-4+\sqrt{19}$, the sufficient condition given in Theorem 1 is not met.

2. As noted earlier, $\Phi_{0}$ does not preserve the class $S[5]$. Thus, we know that there exists a univalent polynomial $p$, such that $\Phi_{0}(p) \notin S$. We've also noted that it is an open problem as to whether $\Phi_{\gamma}\left(P_{n}\right) \subset S$ for $\gamma>0$ and all $n=1,2, \ldots$. The sufficient condition of univalence of $\Phi_{\gamma}\left(P_{n}\right)$ not being met for $n=7$ suggests that we try to show that $\Phi_{0}\left(P_{7}\right) \nsubseteq P_{7}$. It is natural to consider the polynominals

$$
p(z ; n ; j)=z+\sum_{k=2}^{n}\left(\frac{n-k+1}{n} \cdot \frac{\sin (k j \pi /(n+1))}{\sin (j \pi /(n+1))}\right) z^{k}
$$


which were shown to be univalent in $\Delta$ by Suffridge [13]. On the other hand, there are reasons for doubting that $\Phi_{\gamma}(p(z ; 7 ; j)) \notin S$ for $j=1,2, \ldots, 7$. In particular, it can be shown directly that for $\Phi_{\gamma}(p(z ; 7 ; j))=z+\sum_{k=2}^{7} b_{j, k} z^{k}$,

$$
\left|b_{j, k}+b_{j, 8-k}\right| \leqslant\left(1+b_{j, 7}\right) \cdot \frac{\sin (k \pi / 8)}{\sin (\pi / 8)}, \quad(k=2,3, \ldots, 7)
$$

for each $j=1,2, \ldots, 7$ and for all $\gamma>-1$. This set of coefficient conditions was shown in $[13]$ to be necessary for univalence. In addition, we have used a symbolic manipulation program and the Schur-Cohn Criteria to verify that, for $j=1,2, \ldots, 7$, the derivative of each $\Phi_{\gamma}(p(z ; 7 ; j))$ is nonzero in $\Delta$ for $\gamma=0,1,2, \ldots, 15$. Since neither of the conditions is sufficient for univalence, this leaves us with the following

Open Problem 1. Find a univalent polynomial of degree $7, p$, such that $\Phi_{\gamma}(p)$ is not univalent for some $\gamma>-1$.

3. Since for $q_{n} \in P_{n}, \lim _{\gamma \rightarrow \infty} \Phi_{\gamma}\left(q_{n}\right)=q_{n} \in P_{n}$, and $(\gamma+1) /(\gamma+n)<1$, it is also natural to pose

Open Problem 2. For $\gamma$ large enough, show that $\Phi_{\gamma}\left(P_{n}\right) \subset P_{n}$ for all $n$.

\section{THE OPERATOR $\Omega(a, b, c ; \cdot)$.}

Using a method due to Khokhlov [3], we obtain:

Theorem 2. A necessary and sufficient condition such that $\Omega\left(a, b, c ; T^{\star}(\alpha)\right) \subset$ $T^{\star}(\alpha)$ is that $a>0, b>0, c>a+b$ and $\Gamma(c-a-b) \Gamma(c) \leqslant 2 \Gamma(c-a) \Gamma(c-b)$.

Proof: For $f(z)=z-\sum_{n=2}^{\infty} a_{n} z^{n} \in T^{\star}(\alpha)$, let

$$
g(z)=\Omega(a, b, c ; f)=z-\sum_{n=2}^{\infty} d_{n} z^{n}
$$

where $d_{n}=\left((a)_{n-1}(b)_{n-1}\right) /\left((c)_{n-1}(1)_{n-1}\right) \cdot a_{n} \geqslant 0$. From $(1), g \in T^{\star}(\alpha)$ if and only if $\sum_{n=2}^{\infty}((n-\alpha) /(1-\alpha))\left|d_{n}\right| \leqslant 1$. We also know $[10]$ that $\left|a_{n}\right| \leqslant \frac{1-\alpha}{n-\alpha}$. Thus,

$$
\sum_{n=2}^{\infty}\left(\frac{n-\alpha}{1-\alpha}\right)\left|d_{n}\right| \leqslant \sum_{n=2}^{\infty}\left(\frac{(a)_{n-1}(b)_{n-1}}{(c)_{n-1}(1)_{n-1}}\right)=\left\{1+\sum_{n=1}^{\infty}\left(\frac{(a)_{n}(b)_{n}}{(c)_{n}(1)_{n}}\right)\right\}-1
$$

It is well-known [14] that $F(a, b, c ; z)$ is convergent in $\Delta$ for $c>a+b$ and $F(a, b, c ; 1)=\Gamma(c-a-b) \Gamma(c) /(\Gamma(c-a) \Gamma(c-b))$. Therefore, for $c>a+b$, we have $\sum_{n=2}^{\infty}((n-\alpha) /(1-\alpha))\left|d_{n}\right| \leqslant 1$ if and only if $(\Gamma(c-a-b) \Gamma(c) / \Gamma(c-a) \Gamma(c-b))-1 \leqslant$ 1 . 
Remark. For $c \geqslant 3$, we note that $\Omega\left(1,1, c ; T^{\star}(\alpha)\right) \subset T^{\star}(\alpha)$. Therefore, for $n \geqslant 2$, the generalised Biernacki operators

$$
n ! z^{1-n} \int_{0}^{z} \int_{0}^{\tau_{n}} \cdots \int_{0}^{\tau_{2}} \frac{f\left(\tau_{1}\right)}{\tau_{1}} d \tau_{1} \ldots d \tau_{n}
$$

preserve the class $T^{\star}(\alpha)$.

\section{REFERENCES}

[1] M. Biernacki, 'Sur l'integral des fonctions univalentes', Bull. Acad. Polon. Sci., Ser. Math. Astron. Phys. 8 (1980), 29-34.

[2] J.L. Frank, 'Subordination and convex univalent polynomials', J. Reine Angew. Math. 280 (1977), 63-69.

[3] Y.E. Khokhlov, 'Convolutory operators preserving univalent functions', Ukrainian Math. J. 37 (1985), 220-226.

[4] J. Krzyz, 'The radius of close-to-convexity within the family of univalent functions', Bull. Acad. Polon. Sci, Ser. Math. Astron. Phys. 10 (1962), 201-204.

[5] J. Krzyz and $Z$. Lewandowski, 'On the integral of univalent functions', Bull. Acad. Polon. Sci., Ser. Math. Astron. Phys. 11 (1963), 447-448.

[6] J.Krzyz and I. Rahman, 'Univalent polynomials of small degree', Ann. Univ. Mariae CurieSklodowska Sect. A 21 (1967), 79-90.

[7] M. Marden, Geometry of Polynomials, Amer. Math. Soc. Surveys, No. 3, 1963.

[8] St. Ruscheweyh, 'New criteria for univalent functions', Proc. Amer. Math. Soc. 49 (1975), 109-115.

[8] St. Ruscheweyh and T. Sheil-Small, 'Hadamard products of schlicht functions and the PolyaSchoenberg conjecture', Comment. Math. Helv. 48 (1973), 119-135.

[10] H. Silvernan, 'Univalent functions with negative coefficients', Proc. Amer. Math. Soc 51 (1975), 109-116.

[11] H. Silverman and E. Silvia, 'Univalence preserving operators', Complex Variables Theory Appl. 5 (1986), 313-321.

[12] H. Silverman and M. Ziegler, 'Functions of positive real part with negative coefficients', Houston J. Math. (2) 4 (1978), 269-275.

[13] T.J. Suffridge, 'On univalent polynomials', J. London Math. Soc. 44 (1969), 496-504.

[14] E.T. Wittaker and G.N. Watson, $A$ Course of Modern Analysis, 4th edition reprinted (Cambridge University Press, Cambridge, 1980).

\footnotetext{
Department of Mathematics, University of California, Davis, Davis, CA 95616

United States of America.
} 